Supplement of Hydrol. Earth Syst. Sci., 20, 4237-4264, 2016

http://www.hydrol-earth-syst-sci.net/20/4237/2016/

doi:10.5194/hess-20-4237-2016-supplement

(C) Author(s) 2016. CC Attribution 3.0 License.

(c) (1)

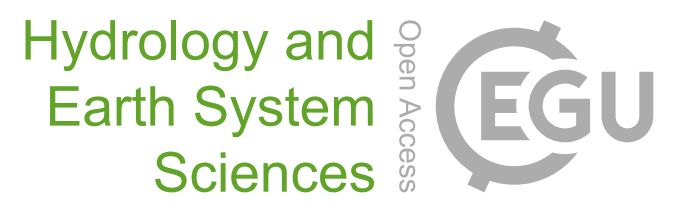

Supplement of

\title{
Canopy-scale biophysical controls of transpiration and evaporation in the Amazon Basin
}

\section{Kaniska Mallick et al.}

Correspondence to: Kaniska Mallick (kaniska.mallick@gmail.com) and Ivonne Trebs (ivonne.trebs@list.lu)

The copyright of individual parts of the supplement might differ from the CC-BY 3.0 licence. 


\section{S1. Derivations of evaporative fraction $(\Lambda)$ 'state equation' in STIC1.2}

2 In order to express $\Lambda$ in terms of $g_{A}$ and $g_{C}$, we had adopted the Advection-Aridity (AA)

3 hypothesis (Brutsaert and Stricker, 1979) with a modification introduced by Mallick et al.

4 (2015). Although the AA hypothesis leads to an assumed link between $g_{A}$ and $T_{0}$, the effects

5 of surface moisture (or water stress) were not explicit in the AA equation. Mallick et al.

6 (2015) implemented a moisture constraint in the original AA hypothesis for deriving an

7 expression of $\Lambda$. A modified form of the original advection-aridity hypothesis is written as 8 follows.

$$
E_{P M}^{*}=2 E_{P T}^{*}-E
$$

9 Here $E_{P M}^{*}$ is the potential evapotranspiration according to Penman-Monteith (Monteith, 1965)

10 for any surface, and $E_{P T}^{*}$ is the potential evapotranspiration according to Priestley-Taylor

11 (Priestley and Taylor, 1972). Dividing both sides by $E$ we get,

$$
\frac{E}{E_{P M}^{*}}=\frac{E}{2 E_{P T}^{*}-E}
$$

12 and dividing the numerator and denominator of the right hand side of eqn. (S2) by $E_{P T}^{*}$ we 13 get,

$$
\frac{E}{E_{P M}^{*}}=\frac{\frac{E}{E_{P T}^{*}}}{2-\frac{E}{E_{P T}^{*}}}
$$

14 Again assuming the Priestley-Taylor equation for any surface is a variant of the PM potential evapotranspiration equation, we will derive an expression of $E_{P T}^{*}$ for any surface.

$$
E_{P M}^{*}=\frac{s \phi+\rho c_{P} g_{A} D_{A}}{s+\gamma\left(1+\frac{g_{A}}{g_{C \max }}\right)}
$$




$$
\begin{aligned}
& =\frac{s \phi}{s+\gamma\left(1+\frac{g_{A}}{g_{C \max }}\right)}\left(1+\frac{\rho c_{P} g_{A} D_{A}}{s \phi}\right) \\
& =\frac{\alpha s \phi}{s+\gamma\left(1+\frac{g_{A}}{g_{C \max }}\right)} \\
& =E_{P T}^{*}
\end{aligned}
$$

1 Here $\gamma$ is the psychrometric constant $\left(\mathrm{hPa} \mathrm{K}^{-1}\right), s$ is the slope of the saturation vapor pressure

2 versus air temperature $\left(\mathrm{hPa} \mathrm{K}^{-1}\right), \alpha$ is the Priestley-Taylor parameter $(\alpha=1.26$ under non-

3 limiting moisture conditions), $D_{A}$ is the vapor pressure deficit of air (hPa). $g_{C \max }$ is defined as

4 the maximum possible $g_{C}$ under the prevailing atmospheric conditions whereas $g_{C}$ is limited

5 due to the moisture availability $(M)$ and hence $g_{C \max }=g_{C} / M$ (Monteith, 1995; Raupach,

6 1998). We assume that $M$ is a significant controlling factor for the ratio of actual and

7 potential evapotranspiration (or transpiration for a dry canopy), and the interactions between

8 the land and environmental factors are substantially reflected in $M$. Since, Penman (1948)

9 derived his equation over the open water surface and $g_{C \max }$ over the water surface is very high

10 (Monteith, 1965; 1981), $g_{A} / g_{C \max }$ was assumed to be negligible.

11 Expressing $\phi$ as $\phi=E / \Lambda$ and expressing $E_{P T}^{*}$ according to eqn. (S5) gives the following 12 expression of $E / E_{P T}^{*}$.

$$
\frac{E}{E_{P T}^{*}}=\frac{\Lambda\left[s+\gamma\left(1+\frac{g_{A}}{g_{C \max }}\right)\right]}{\alpha s}
$$

13 Now substituting $E / E_{P T}^{*}$ from eqn. (S6) into eqn. (S3) and after some algebra we obtain the 14 following expression.

$$
\frac{E}{E_{P M}^{*}}=\frac{\Lambda\left[s+\gamma\left(1+\frac{g_{A}}{g_{C \max }}\right)\right]}{2 \alpha s-\Lambda\left[s+\gamma\left(1+\frac{g_{A}}{g_{C \max }}\right)\right]}
$$


1 According to the PM equation (Monteith, 1965) of actual and potential evapotranspiration,

$$
\frac{E}{E_{P M}^{*}}=\frac{\frac{s \phi+\rho c_{p} g_{A} D_{A}}{s+\gamma\left(1+\frac{g_{A}}{g_{C}}\right)}}{\frac{s \phi+\rho c_{p} g_{A} D_{A}}{s+\gamma\left(1+\frac{g_{A}}{g_{C \max }}\right)}}
$$

2 Combining eqn. (S7) and (S8) (eliminating $E / E_{P M}^{*}$ ) gives an expression for $\Lambda$ in terms of the 3 conductances.

$$
\frac{s+\gamma\left(1+\frac{M g_{A}}{g_{C}}\right)}{s+\gamma\left(1+\frac{g_{A}}{g_{C}}\right)}=\frac{\Lambda\left[s+\gamma\left(1+\frac{M g_{A}}{g_{C}}\right)\right]}{2 \alpha s-\Lambda\left[s+\gamma\left(1+\frac{M g_{A}}{g_{C}}\right)\right]}
$$

4 After some algebra the final expression of $\Lambda$ is as follows.

$$
\Lambda=\frac{2 \alpha s}{2 s+2 \gamma+\gamma \frac{g_{A}}{g_{C}}(1+M)}
$$

